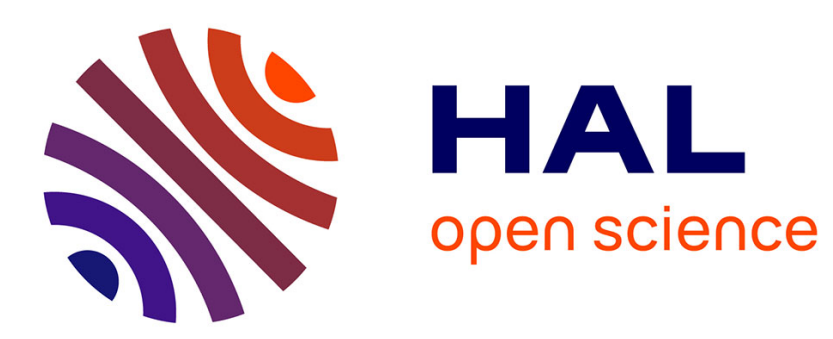

\title{
On some theoretical and computational aspects of Anatol Vieru's periodic sequences
}

Moreno Andreatta, Dan Tudor Vuza, Carlos Agon

\section{To cite this version:}

Moreno Andreatta, Dan Tudor Vuza, Carlos Agon. On some theoretical and computational aspects of Anatol Vieru's periodic sequences. Soft Computing, 2004, 8 (9), pp.588-596. hal-01161049

\author{
HAL Id: hal-01161049 \\ https://hal.science/hal-01161049
}

Submitted on 8 Jun 2015

HAL is a multi-disciplinary open access archive for the deposit and dissemination of scientific research documents, whether they are published or not. The documents may come from teaching and research institutions in France or abroad, or from public or private research centers.
L'archive ouverte pluridisciplinaire HAL, est destinée au dépôt et à la diffusion de documents scientifiques de niveau recherche, publiés ou non, émanant des établissements d'enseignement et de recherche français ou étrangers, des laboratoires publics ou privés. 


\title{
On some theoretical and computational aspects of Anatol Vieru's periodic sequences
}

\author{
Moreno Andreatta ${ }^{1}$, Dan Tudor Vuza ${ }^{2}$, Carlos Agon ${ }^{1}$ \\ 1 Ircam-CNRS, e-mail: andreatta@ircam.fr e-mail: agonc@ircam.fr \\ 2 The Institute of Mathematics of the Romanian Academy, e-mail: Dan.Vuza@imar.ro
}

01 May 2004

\begin{abstract}
This article develops some aspects of Anatol Vieru's compositional technique based on finite difference calculus of periodic sequences taking values in a cyclic group. After recalling some group-theoretical properties, we focus on the decomposition algorithm enabling to represent any periodic sequence taking values in a cyclic group as a sum of a reducible and a reproducible sequence. The implementation of this decomposition theorem in a computer aided music composition language, as OpenMusic [1] , enables to easily verify if a given periodic sequence is reducible or reproducible. In this special case, one of the two terms will be identically zero. This means that every periodic sequence has in itself a certain degree of reducibility and reproducibility. We also suggest how to use this result in order to explain some regularities of the distribution of numerical values in the case of the finite addition process and how to generalize the decomposition theorem by means of the Fitting Lemma. This opens the problem of the musical relevance of a generalized module-theoretical approach in Vieru's theory of periodic sequences.
\end{abstract}

\section{Introduction}

The theory of periodic sequences has a very important place in the modal universe of the Romanian composer Anatol Vieru (1926-1998). This theory, which has been originally described by Vieru in his Book of Modes [7], is based - according to him - on one of the most enigmatic dichotomy existing in music, i.e., the duality between sounds and intervals. This duality is expressed, although implicitly, by the group-theoretical formalization of the well-tempered system by assuming that a given cyclic group of order $n$ may represent, at the same time, the collection of pitches (or pitch classes) and intervals (or interval classes). The finite difference calculus applied to periodic sequences taking values in a cyclic group enables the composer to explore this duality iteratively.
For example, by interpreting a given periodic sequence in the pitch domain, the first layer of the finite difference process transforms the previous musical structures in the ordered collection of its intervals. By iterating the finite difference calculus in the new sequence, the intervallic relationships between the intervals themselves clearly appear and so on, with the possibility of interpreting any new periodic sequence again as a pitch content sequence or as an intervallic sequence. In the next section of this paper we give the formal definition of the finite difference process and we describe two families of periodic sequences, the reducible and the reproducible sequences. Their relationships are established by a decomposition theorem that we originally introduced in a previous paper [3] and that we will describe here in more details by also proposing some generalization via the Fitting Lemma. The final section discusses an application of the previous result for a phenomenon of proliferation of some very special values inside the derivatives of an initial periodic sequence by the dual process of the finite difference calculus: the addition process (see [2] for a more general discussion on the musical relevance of algebraic methods).

\section{Periodic Sequences taking values in a cyclic group}

\subsection{Basic definitions}

Definition 1 A periodic sequence is a map $f$ defined on $\mathbf{Z}$ taking values in the cyclic group $\mathbf{Z}_{n}$ such that there exists an integer $m$ satisfying the equation $f(x+m)=$ $f(x)$ for any $x \in \mathbf{Z}$.

Several musical interpretations can be associated with a periodic sequence. In figure 1, the sequence:

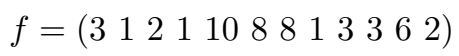

denotes a periodic rhythm, where each element of $f$ is a multiple of a eigth-note. 


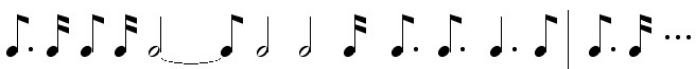

Fig. 1 The sequence $f$ interpreted as a periodic rhythmic pattern

Figure 2 shows the same sequence, but this time interpreted as a melody, where each element of $f$ is associated with a note in a scale of semitones starting from C4.

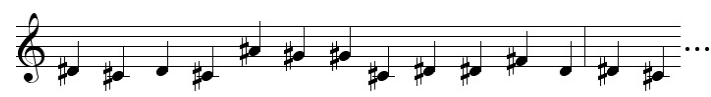

Fig. 2 The sequence $f$ interpreted as a periodic melodic pattern

In figure 3 , the elements of $f$ are taken as intervals between two consecutive notes.

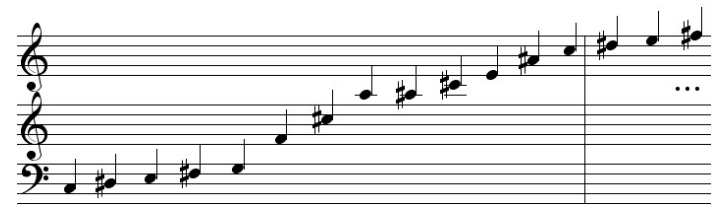

Fig. 3 The sequence $f$ as a periodic intervallic pattern

Definition 2 The translated sequence $T f$ of a (periodic) sequence $f$ is the periodic sequence $T f$ defined by the following equation:

$$
T f(x)=f(x+1)
$$

Definition 3 The sequence of differences of a (periodic) sequence $f$ is the periodic sequence $D f$ defined by the following equation:

$$
D f(x)=f(x+1)-f(x) .
$$

The relationship between the translated sequence and the sequence of differences is expressed by the following equation :

$$
D=T-1 \text {. }
$$

Affirming that $f$ is $m$-periodic is equivalent to the relation $T^{m} f=f$.

Example 1 In the theory of unending rhythmic canons, as originally introduced by one of the authors in [8], a tiling canon can be considered as the factorization of a cyclic group $\mathbf{Z}_{n}$ into two periodic sequences $f$ and $g$. In figure 4 the two subsets $f$ and $g$ are respectively $\{0,8$, $10\}$ and $\{0,5,6,11\}$. Factoring a cyclic group into a direct sum of subsets $f$ and $g$ means that every element of $\mathbf{Z}_{n}$ can be expressed in a unique way as a sum of an element of $f$ and an element of $g$. Musically, this means that at every instant there is one (and only one) voice playing.

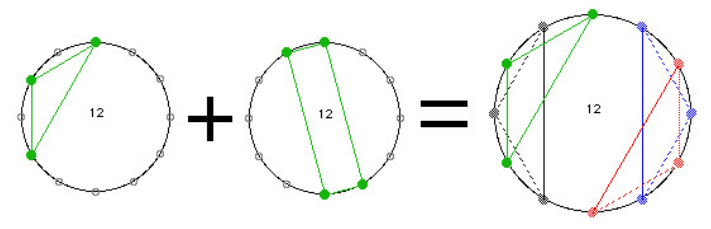

Fig. 4 Factorization of $\mathbf{Z}_{12}$ in two sequences having periods equal to 12 and 6 respectively

Figure 5 shows a canon in 4 voices obtained by the time translation of the pattern $\mathrm{R}=\left(\begin{array}{lll}2 & 8 & 2\end{array}\right)$ in the onsettimes $0,5,6,11$.

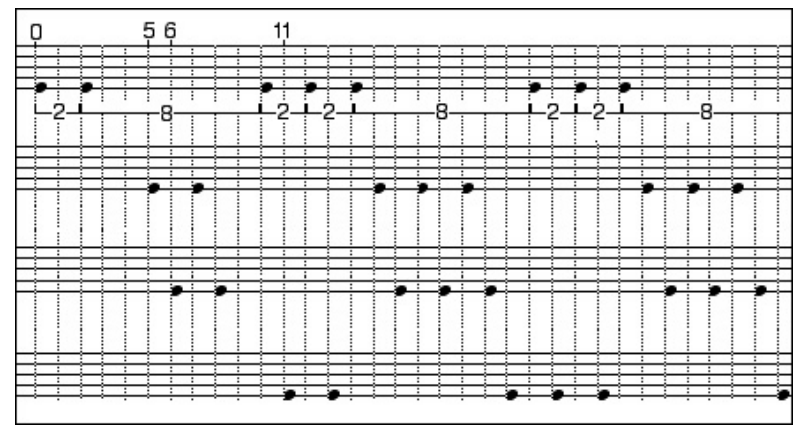

Fig. 5 A tiling rhythmic canon corresponding to the factorization of $\mathbf{Z}_{12}$ into two subsets $f$ and $g$

\subsection{Reducible sequences}

Definition 4 The sequence $f$ is called reducible if there is an integer $k \geq 0$ such that $D^{k} f=0$. We will designate by $\operatorname{Red}(G)$ the family of reducible sequences taking values in a group $G$, i.e., the family of reducible $G$-valued sequences.

Example 2 Let $f=\left(\begin{array}{l}25332\end{array}\right)$ a sequence of period 5 in $\mathbf{Z}_{5}$. By iterating the difference operator 4 times, we end up with the zero sequence:

$$
\begin{aligned}
& D^{1}=\left(\begin{array}{lllll}
3 & 3 & 0 & 4 & 0
\end{array}\right) \\
& D^{2}=\left(\begin{array}{lllll}
0 & 2 & 4 & 1 & 3
\end{array}\right) \\
& D^{3}=\left(\begin{array}{lllll}
2 & 2 & 2 & 2 & 2
\end{array}\right) \\
& D^{4}=\left(\begin{array}{lllll}
0 & 0 & 0 & 0 & 0
\end{array}\right)
\end{aligned}
$$

In figure $6 f$ is interpreted as a sequence of intervals in a chord. For each $D^{i}(f)$ we have a new chord until the trivial case of the final singleton chord is reached.

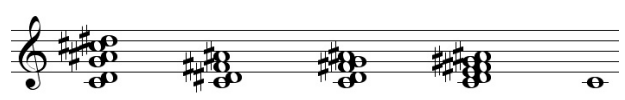

Fig. 6 Reduction of an intervallic sequence interpreted as chords 
Remark 1 More generally, given two integers $m$ and $k$ and a prime number $p$, every sequence of period $p^{m}$ in $\mathbf{Z}_{p^{k}}$ is a reducible sequence. [For a proof by induction on $m$ see [3], p. 5.]

\subsection{Reproducible sequences}

Definition 5 The sequence $f$ is called reproducible if an integer $k \geq 0$ does exist such that $D^{k} f=f$. $\operatorname{Rep}(G)$ will indicate the family of reproducible sequences taking values in a given group $G$.

Example 3 Let $f=(256341)$ be a sequence of period 6 in $\mathbf{Z}_{7}$. This sequence reproduces itself after 6 iterations of the difference operator:

$$
\begin{aligned}
& D^{1}=\left(\begin{array}{llllll}
3 & 1 & 4 & 1 & 4 & 1
\end{array}\right) \\
& D^{2}=\left(\begin{array}{lllllll}
5 & 3 & 4 & 3 & 4 & 2
\end{array}\right) \\
& D^{3}=\left(\begin{array}{llllll}
5 & 1 & 6 & 1 & 5 & 3
\end{array}\right) \\
& D^{4}=\left(\begin{array}{lllllll}
3 & 5 & 2 & 4 & 5 & 2
\end{array}\right) \\
& D^{5}=\left(\begin{array}{lllllll}
2 & 4 & 2 & 1 & 4 & 1
\end{array}\right) \\
& D^{6}=\left(\begin{array}{llllll}
2 & 5 & 6 & 3 & 4 & 1
\end{array}\right)
\end{aligned}
$$

Figure 7 shows the sequence of chords corresponding to the previous difference process.

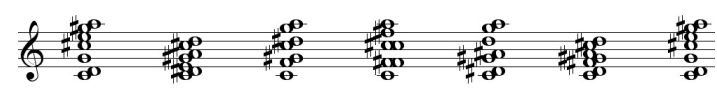

Fig. 7 Reproduction of an intervallic sequence after six iterations of the difference operator

More generally, one can show that every sequence of $p-1$ distinct elements in $\mathbf{Z}_{p}$ is a reproducible sequence [See [3] for some characterization theorems of reproducible sequences.]

\section{Reducible and reproducible sequences as generators}

\subsection{The foundamental decomposition theorem}

The foundamental result of Vieru's theory of periodic sequences states that the two previous families of reducible and reproducible sequences can be considered as sequences generating all periodic sequences. In other words:

Theorem 1 Every periodic sequence $f$ can be decomposed into a unique way as a sum of a reducible and a reproducible sequence, i.e.,:

$$
f=f_{\text {red }}+f_{\text {rep }}, f_{\text {red }} \in \operatorname{Red}(\mathrm{G}), f_{\text {rep }} \in \operatorname{Rep}(\mathrm{G}) .
$$

Proof Let $f$ be $m$ - periodic. The collection of sequences $m$-periodic being finite, there exist two integers $k, l \geq 1$ such that $D^{k} f=D^{k+l} f$. By induction on $r$ one has $D^{k} f=D^{k+r l} f$. In the same way it can be shown that there exist two integers $r, s \geq 1$ such that $D^{r l} f=$ $D^{(r+s) l} f$. We put

$$
f_{\text {red }}=f-D^{r l} f, f_{\text {rep }}=D^{r l} f .
$$

It follows that $D^{k}\left(f-D^{r l} f\right)=0, D^{s l} D^{r l} f=D^{r l} f$, which means that $f_{\text {red }}$ and $f_{\text {rep }}$ give the needed decomposition. The unicity comes from the relation

$$
\operatorname{Red}(\mathrm{G}) \cap \operatorname{Rep}(\mathrm{G})=\{0\}
$$

\subsection{Decomposition of $\mathbf{Z}_{n}$ into $p$-groups}

There is a one-to-one relation between the subgroups of $\mathbf{Z}_{n}$ and the family of integers $d$ that divide $n$ with $1 \leq d \leq n$, i.e., for any such $d$ we may take the unique subgroup of $\mathbf{Z}_{n}$ with $d$ elements. The latter can be characterised as the set of $z \in \mathbf{Z}_{n}$ such that $d z=0$ or, equivalently, as the set $\frac{n}{d} \mathbf{Z}_{n}$ of elements having the form $\frac{n}{d} z$ where $z$ belongs to $\mathbf{Z}_{n}$.

Definition 6 The abelian group $G$ is a direct sum of a family of subgroups $G_{1}, \ldots, G_{m}$ of $G$ if any $x \in G$ can be decomposed in a unique way into a sum $x_{1}+\ldots+x_{m}$ with $x_{i} \in G_{i}$ for $1 \leq i \leq m$. We will write $G=\bigoplus_{i=1}^{m} G_{i}$.

Definition 7 Let $p$ be a prime number. A finite abelian group is called $p$-group if its cardinality is a power of $p$.

Theorem 2 Any group $\mathbf{Z}_{n}$ can be decomposed as the direct sum of its p-maximals subgroups.

If $n=\prod_{i=1}^{m} p^{k_{i}}$ is the decomposition of an integer $n$ into prime factors, the decomposition of $\mathbf{Z}_{n}$ in maximal $p$-subgroups can be written as follows

$$
\mathbf{z}_{n}=\bigoplus_{i=1}^{m} G_{p^{k_{i}}}
$$

where $G_{p^{k_{i}}}$ is the subgroup of $\mathbf{Z}_{n}$ with $p^{k_{i}}$ elements. The decomposition of any $z \in \mathbf{Z}_{n}$ defines in a unique way the elements $\pi_{i}(z) \in G_{p^{k_{i}}}$ such that $z=\sum_{i=1}^{m} \pi_{i}(z)$. The arrows $\pi_{i}: \mathbf{Z}_{n} \rightarrow G_{p^{k_{i}}}$ are group homomorphisms.

The $p_{i}$-component $\pi_{i}(z)$ of $z$ is the unique element $y \in$ $\mathbf{Z}_{n}$ satisfying the relations $p^{k_{i}} y=\frac{n}{p^{k_{i}}}(z-y)=0$ in $\mathbf{Z}_{n}$. Let $q_{i}$ be an integer verifying

$$
q_{i} \frac{n}{p^{k_{i}}}=1 \bmod p^{k_{i}} \text {. }
$$

It follows that $p^{k_{i}} \frac{n}{p^{k_{i}}} q_{i} z=\frac{n}{p^{k_{i}}}\left(z-\frac{n}{p^{k_{i}}} q_{i} z\right)=0$ in $\mathbf{Z}_{n}$, which means that

$$
\pi_{i}(z)=\frac{n}{p^{k_{i}}} q_{i} z
$$


Example 4 All subgroups of $\mathbf{Z}_{12}$ have a natural musical interpretation. Namely:

the unison

$\mathrm{G}_{1}=\{0\}$

the interval of tritone

$\mathrm{G}_{2}=\{0,6\}$

the augmented triad as the maximal 3-group

$\mathrm{G}_{3}=\{0,4,8\}$

the diminished seventh as the maximal 2-group

$\mathrm{G}_{4}=\{0,3,6,9\}$

the whole tone scale

$\mathrm{G}_{6}=\{0,2,4,6,8,10\}$

the chromatic scale

$\mathrm{G}_{12}=\{0,1,2,3,4,5,6,7,8,9,10,11\}$

Example 5 Decomposition of $\mathbf{Z}_{12}$ into $p$-groups

$$
\begin{aligned}
& \mathbf{z}_{12}=G_{3} \bigoplus G_{4} \\
& \begin{array}{l|llll}
8 & 8 & 11 & 2 & 5
\end{array} \\
& q_{3}=1,1 \cdot \frac{12}{3}=1 \bmod 3, \\
& q_{4}=-1,-1 \cdot \frac{12}{4}=1 \bmod 4, \\
& \pi_{3}(x)=\frac{12}{3} q_{3} x=4 x, \pi_{4}(x)=\frac{12}{4} q_{4} x=-3 x . \\
& 5=8+9=4 \cdot 5-3 \cdot 5=4 \cdot 2-3 \cdot 1 \\
& 7=4+3=4 \cdot 7-3 \cdot 7=4 \cdot 1-3 \cdot 3 \\
& 11=8+3=4 \cdot 11-3 \cdot 11=4 \cdot 2-3 \cdot 3
\end{aligned}
$$

\begin{tabular}{l|llll} 
& 0 & 3 & 6 & 9 \\
\hline 0 & 0 & 3 & 6 & 9
\end{tabular}

$\begin{array}{lllll}4 & 4 & 7 & 10 & 1\end{array}$

Remark 2 Many theoretical works (see in particular [4] and [6]) suggest to use the decomposition of the cyclic group $\mathbf{Z}_{12}$ into a direct sum of its maximal $p$-groups, as the natural representation for the tone space. This leads to the toroidal representation shown in figure 8 .

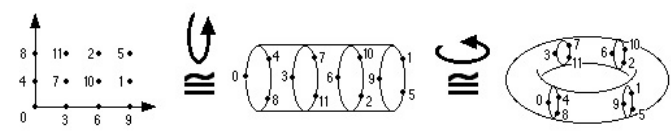

Fig. 8 The toroidal representation of the well-tempered tone space
3.3 Periodised sequences and the calculation of reducible and reproducible components of a periodic sequence

Definition 8 For all $m$-periodic sequences $f$ and for all integer $d$ dividing $m$, the $d$-periodised of $f$ is the sequence of period d expressed by the following relation:

$$
\sum_{k=0}^{m / d-1} T^{k d} f
$$

Proposition 1 Let $f=\sum_{j} f_{j}$ be the decomposition of the periodic sequence $f$ corresponding to the decomposition of $\mathbf{Z}_{n}$ in $p$-groups and let $f_{j}=f_{j, \text { red }}+f_{j, \text { rep }}$ be the decomposition of $f_{j}$ in a sum of a reducible and a reproducible sequence. Then the decomposition of $f$

$$
f=f_{\text {red }}+f_{\text {rep }}, f_{\text {red }} \in \text { Red, } f_{\text {rep }} \in \text { Rep }
$$

is given by

$$
f_{\text {red }}=\sum_{j} f_{j, r e d}, f_{\text {rep }}=\sum_{j} f_{j, r e p} .
$$

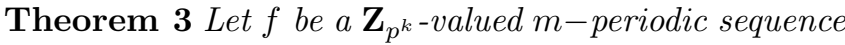
and let $f_{\text {per }}$ be the $p^{r}$-periodised of $f$, where $p^{r}$ is the highest power of $p$ dividing $m$. Let $\left(\frac{m}{p^{r}}\right)^{-1}$ be the inverse of $\frac{m}{p^{r}} \bmod p^{k}$. Then the decomposition of $f$

$$
f=f_{\text {red }}+f_{\text {rep }}, f_{\text {red }} \in \text { Red, } f_{\text {rep }} \in \text { Rep }
$$

is given by

$$
f_{\text {red }}=\left(\frac{m}{p^{r}}\right)^{-1} f_{\text {per }}, f_{\text {rep }}=f-\left(\frac{m}{p^{r}}\right)^{-1} f_{\text {per }} .
$$

Proof Since $f_{\text {red }}$ is $p^{r}$-periodic by construction, it is reducible from a previous remark on the reproducibility of any $p^{m}$-periodic sequence in $\mathbf{Z}_{p^{k}}$. The $p^{r}$-periodised of $f_{\text {rep }}$ is zero since:

$$
\begin{gathered}
\sum_{i=0}^{m / p^{r}-1} T^{i p^{r}} f_{r e p}= \\
=\sum_{i=0}^{m / p^{r}-1} T^{i p^{r}} f-\left(\frac{m}{p^{r}}\right)\left(\frac{m}{p^{r}}\right)^{-1} f_{\text {per }}= \\
=f_{\text {per }}-f_{\text {per }}=0
\end{gathered}
$$

The reproducibility of $f_{\text {rep }}$ comes from a general result for $\mathbf{Z}_{p^{k}}$-valued $m$-periodic sequences $f$ stating that $f$ is reproducible if and only if the $p^{r}$-periodised of $f$ is zero, where $p^{r}$ is the highest power of $p$ that divides $m$. [For a proof of this result, see [3], p. 7].

Corollary 1 Let $m$ and $n$ be two integers such that $m \wedge$ $n=1$ and let $f$ be a $\mathbf{Z}_{n}$-valued $m$-periodic sequence. $f$ is reproducible if and only if $\sum_{i=0}^{m-1} f(i)=0$. 


\subsection{The decomposition algorithm}

We now describe step by step how to obtain the decomposition of a $\mathbf{Z}_{n}$-valued periodic sequence $f$.

1. Write the decomposition of $n$ in prime factors:

$$
n=\prod_{j=1}^{N} p_{j}^{k_{j}}
$$

2. Find the integers $q_{j}$ such that

$$
q_{j} \frac{n}{p_{j}^{k_{j}}}=1 \bmod p_{j}^{k_{j}}
$$

3. For all $j$ construct the sequences $f_{j, r e d}$ and $f_{j, r e p}$ as follows:

3.1 Set $f_{j}(x)=\varphi_{j}(f(x))$, where $\varphi_{j}: \mathbf{Z}_{n} \rightarrow \mathbf{Z}_{p_{j}^{k_{j}}}$ is the canonic map.

3.2 Let $p_{j}^{r_{j}}$ be the highest power of $p_{j}$ dividing the period $m$ of $f$. Determine the inverse $\left(\frac{m}{p_{j}^{r_{j}}}\right)^{-1}$ of $\frac{m}{p_{j}^{r_{j}}} \bmod p_{j}^{k_{j}}$.

3.3 Write down the $m$ elements of the period of $f_{j}$ as a table with $\frac{m}{p_{j}^{r_{j}}}$ lines and $p_{j}^{r_{j}}$ columns (if $r_{j}=0$, the

table will have a unique column). Add a line given by the elements of any column and by multiplying by $\left(\frac{m}{p_{j}^{r_{j}}}\right)^{-1}$ module $p_{j}^{k_{j}}$.

3.4 Let $f_{j, r e d}$ be the $p_{j}^{r_{j}}$ - periodic sequence defined by the line constructed at the previous step and construct $f_{j, r e p}=f_{j}-f_{j, r e d}$.

4 By setting

$$
f_{\text {red }}=\sum_{i=1}^{N} q_{j} \frac{n}{p_{j}^{k_{j}}} f_{j, r e d}, f_{\text {rep }}=\sum_{i=1}^{N} q_{j} \frac{n}{p_{j}^{k_{j}}} f_{j, \text { rep }} .
$$

we have the decomposition of any periodic sequence in a reducible and reproducible component.

Example 6 Consider the following $\mathbf{Z}_{n}$-valued sequence $f=\left(\begin{array}{llllllllll}0 & 0 & 7 & 4 & 4 & 3 & 34 & 4 & 7\end{array}\right)$. By applying the previous algorithm we have:

$$
\begin{aligned}
& \text { 1. } 12=2^{2} .3^{1}, N=2, p_{1}^{k_{1}}=4 \text { and } p_{2}^{k_{2}}=3 \\
& \text { 2. } q_{1}=1 \text { and } q_{2}=3 \\
& 3.1 f_{1}=\left(\begin{array}{llllllllllll}
0 & 0 & 3 & 3 & 0 & 0 & 3 & 3 & 0 & 0 & 3 & 0
\end{array}\right) \\
& f_{2}=\left(\begin{array}{llllllllllll}
0 & 0 & 1 & 1 & 1 & 1 & 0 & 0 & 1 & 1 & 1 & 1
\end{array}\right) \\
& 3.2 p_{1}^{r_{1}}=2^{2} \text { and } p_{2}^{r_{2}}=3^{1}
\end{aligned}
$$

\begin{tabular}{lccc} 
& 0 & 0 & 1 \\
& 1 & 1 & 1 \\
& 0 & 0 & 1 \\
& 1 & 1 & 1 \\
\cline { 2 - 4 } & 2 & 2 & 1 \\
$\div 4 \bmod 3$ & 2 & 2 & 1 \\
$\times 4 \bmod 12$ & 8 & 8 & 4
\end{tabular}

\begin{tabular}{lcccc} 
& 0 & 0 & 3 & 3 \\
& 0 & 0 & 3 & 3 \\
& 0 & 0 & 3 & 3 \\
\cline { 2 - 5 } & 0 & 0 & 1 & 1 \\
$\div 3 \bmod 4$ & 0 & 0 & 3 & 3 \\
$\times-3 \bmod 12$ & 0 & 0 & 3 & 3
\end{tabular}

$$
\begin{aligned}
& f_{1}=\left(\begin{array}{lllllllllll}
0 & 0 & 3 & 3 & 0 & 0 & 3 & 3 & 0 & 0 & 3
\end{array}\right)
\end{aligned}
$$

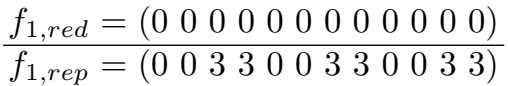

$$
\begin{aligned}
& f_{2}=\left(\begin{array}{llllllllllll}
0 & 0 & 1 & 1 & 1 & 1 & 0 & 0 & 1 & 1 & 1 & 1
\end{array}\right)
\end{aligned}
$$

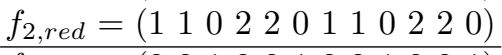

$$
\begin{aligned}
& f_{2, \text { rep }}=\left(\begin{array}{lllllllll}
2 & 21221221221
\end{array}\right)
\end{aligned}
$$

4 .

$$
\begin{aligned}
& f_{\text {red }}=\left(\begin{array}{lllllll}
8 & 8 & 7118411 & 1148 & 41 & 7
\end{array}\right)
\end{aligned}
$$

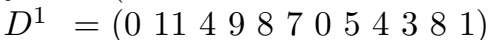

$$
\begin{aligned}
& D^{2}=\left(\begin{array}{lllll}
11 & 5511 & 115511 & 5155 & 51
\end{array}\right) \\
& D^{3}=\left(\begin{array}{llllllllllll}
6 & 0 & 6 & 0 & 6 & 0 & 6 & 0 & 6 & 0 & 6 & 0
\end{array}\right)
\end{aligned}
$$

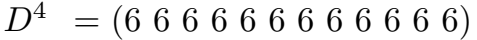

$$
\begin{aligned}
& D^{5}=\left(\begin{array}{llllllllllll}
0 & 0 & 0 & 0 & 0 & 0 & 0 & 0 & 0 & 0 & 0 & 0
\end{array}\right) \\
& f_{\text {rep }}=\left(\begin{array}{lllllllll}
4 & 0880440880
\end{array}\right) \\
& D^{1}=\left(\begin{array}{lllllllllll}
0 & 8 & 8 & 0 & 4 & 4 & 0 & 8 & 8 & 0 & 4
\end{array}\right) \\
& D^{2}=\left(\begin{array}{llllllllll}
8 & 044 & 0 & 8 & 804408
\end{array}\right) \\
& D^{3}=(440880440880)
\end{aligned}
$$

3.5 Towards a generalized version of the decomposition theorem

As originally suggested by mathematician Guerino Mazzola in a private communication with some of the authors, the decomposition theorem is a special case of a more general result known as the Fitting Lemma. In the case of a group $G$, the Fitting Lemma can be expressed in this way. Let $M$ be a family of endomorphisms on $G$ such that $G$ satisfies both descending and ascending chain conditions on normal $M$-subgroups and let $D$ be a normal $M$-endomorphism of $G$. Then $G$ can be expressed as the direct product of two normal subgroups $H$ and $K$ where $D(H)=H$ and $K$ is the kernel of $D$ (i.e., $k \in K$ if and only if there is some integer $a$ such that $D^{a}(k)=e$ where $e$ is the identity of the group $\left.G\right)$. This result can be generalized since the Fitting Lemma holds for any module of finite length. Let $G(m, \mathbf{Z})$ be the set of $m$-periodic $G$-valued sequences. This is a subspace of the space $G(\mathbf{Z})$ of sequences taking values in the group $G$. $G(\mathbf{Z})$ is a $\mathbf{Z}$-module so, thanks to the Fitting Lemma, there exist a power $k$ of the $D$ operator and there exist two sub-modules $U$ and $V$ of $G(m, \mathbf{Z})$ such that $U=\operatorname{Ker}\left(D^{k}\right)$ and the restriction of $D^{k}$ to the sub-module $V$ is an automorphism of $V$. By definition $\operatorname{Ker}(D)$ coincides with the set $\operatorname{Red}_{G}(m, \mathbf{Z})$ of reducible periodic sequences taking values in $G$. For what concerns the sub-module $V$, the previous condition is in general not as strong as the reproducibility condition. This naturally leads to a new concept: the quasi-reproducible sequence. 
Definition 9 A sequence is called quasi-reproducible if there exist a sub-module of $G(m, \mathbf{Z})$ and an integer $k$ such that the restriction of $D^{k}$ to this sub-module is an automorphism.

If the module is finite, there must exist an integer $s$ such that the restriction of $\left(D^{k}\right)^{s}$ to the sub-module is the identity. Without loss of generality, one can take an integer $k$ in such a way that $G(m, \mathbf{Z})$ is the direct sum of $\operatorname{Ker}\left(D^{k}\right)$ and $\operatorname{Rep}(G(m, \mathbf{Z}))$ where:

1. $\operatorname{Ker}\left(D^{k}\right)$ is by definition the module $\operatorname{Red}(G(m, \mathbf{Z}))$ of reducible periodic sequences

2. $\operatorname{Rep}(G(m, Z))$ is the module of reproducible periodic sequences

In conclusion, the decomposition theorem could be stated in the following generalized version:

Theorem 4 (Generalized Decomposition Theorem). Every periodic sequence taking values in a finite group (respectively in a module of finite length) can be decomposed in a unique way as a sum of a reducible and of a reproducible (respectively quasi-reproducible) sequence.

Remark 3 This new version of the decomposition theorem opens up the problem of the musical relevance of the generalization process in music theory. In the case of a module with finite length, the Fitting Lemma does not offer the way of obtaining the decomposition. Moreover, the concept of quasi-reproducibility, which has been introduced for modules with finite length but non finite if considered as sets, seems too abstract to have any natural musical application. Take, for example, the periodic sequences taking values on a subfield $K$ of the field $\mathbf{C}$ of complex numbers, like $\mathbf{Q}, \mathbf{R}$ or $\mathbf{C}$ itself. Here, $K$ is considered as a module on itself. By using Fourier transform, as originally introduced by one of the authors in [8], it is possible to show that the only reducible sequences are the constant ones. Moreover, each sequence $g$ that can be written as $D f$. Then quasi-reproducibility is equivalent to:

$$
\sum_{x=1}^{p} g(x)=0
$$

where $p$ is the period of $g$. The generalized decomposition theorem states that any $K$-valued periodic sequence can be decomposed into a sum of a constant sequence and of a sequence verifying the previous property. The musical relevance of this result is still an open question.

Remark 4 According to David Lewin, one could use the Fitting Lemma to obtain the decomposition in the case of some very particular $d$-periodic sequences $f=\left(x_{1} x_{2} \ldots x_{d}\right)$ taking values in $\mathbf{Z}_{n}$ and such that:

$$
\sum_{i=1}^{d} f\left(x_{i}\right)=n
$$

We will discuss this new decomposition strategy with a simple example. Take the following 3 -periodic sequence:

$$
f=\left(\begin{array}{lll}
5 & 3 & 4
\end{array}\right)
$$

If we iterate the difference operator $D$ we obtain:

$$
\begin{aligned}
& D^{1}=\left(\begin{array}{lll}
10 & 1 & 1
\end{array}\right) \\
& D^{2}=\left(\begin{array}{lll}
3 & 0 & 9
\end{array}\right) \\
& D^{3}=\left(\begin{array}{lll}
9 & 9 & 6
\end{array}\right) \\
& D^{4}=\left(\begin{array}{lll}
0 & 9 & 3
\end{array}\right) \\
& D^{5}=\left(\begin{array}{lll}
9 & 6 & 9
\end{array}\right) \\
& D^{6}=\left(\begin{array}{lll}
9 & 3 & 0
\end{array}\right) \\
& D^{7}=\left(\begin{array}{lll}
6 & 9 & 9
\end{array}\right) \\
& D^{8}=\left(\begin{array}{lll}
3 & 0 & 9
\end{array}\right)
\end{aligned}
$$

We see that the sequence $\left(\begin{array}{lll}3 & 0 & 9\end{array}\right)$ is its own sixth derivative. So, every sequence in the loop

$$
\left(D^{2}(f) D^{3}(f) \ldots D^{7}(f)\right)
$$

is its own sixth derivative. Now, because $D^{6}(f)=\left(\begin{array}{lll}9 & 3 & 0\end{array}\right)$ is in the loop, $D^{6}(f)$ is also the sixth derivative of itself.

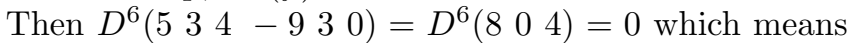
that $(804)$ is in $\operatorname{Ker}\left(D^{k}\right)$ i.e., the sequence $(804)$ is reducible. Thus $\left(\begin{array}{lll}5 & 3 & 4\end{array}\right)=\left(\begin{array}{lll}9 & 3 & 0\end{array}\right)+\left(\begin{array}{lll}8 & 0 & 4\end{array}\right)$ where $(930)$ and ( $\left.8 \begin{array}{ll}8 & 0\end{array}\right)$ are respectively the reducible and reproducible components of the sequence $f$. See [5] for more examples on group-theoretical methods in American Transformational Theory.

\section{Proliferation of some values in a periodic sequence}

Definition 10 Given an element $c_{0} \in \mathbf{Z}_{n}$, the sequence of successive additions of a periodic sequence $f$ is the periodic sequence Af defined by the following equation:

$$
A f(x)= \begin{cases}c_{0} & \text { if } x=0, \\ f(x-1)+A f(x-1) & \text { otherwise. }\end{cases}
$$

where, $c_{0}$ is the starting value of the sequence.

Anatol Vieru made use of the successive addition process in several pieces. In the piece Zone d'oubli (1973), the sequence $f=\left(\begin{array}{llll}6 & 6 & 6 & 6\end{array} 6\right)$ corresponding to the interval of triton is taken as the starting point of the additive process. By successive additions and choosing an arbitrary $c_{i}$ at each addition, Vieru generates ten new sequences that he calls levels. The first initial value is $c_{0}=7$ and this gives the following 2-periodic sequence as the first level:

$$
A f=\left(\begin{array}{llllll}
7 & 1 & 7 & 1 & 7 & 1
\end{array}\right)
$$

The second level starts with $c_{1}=10$ and it has period 6 , and so on until the tenth level of period 864 is reached.
0

I

II

$\ldots$

$\mathrm{X}$ a sequence of period 864

66666

$\begin{array}{llllll}7 & 1 & 7 & 1 & 7 & 1\end{array}$

$\begin{array}{lllllll}10 & 5 & 6 & 1 & 2 & 9 & 10\end{array}$ 
In the case of Zone d'oubli, each level is mapped with a different musical parameter. In particular, the level $\mathrm{V}$ corresponds to the pitch-parameter, with the usual Creferential system (i.e., $\mathrm{C}=0, \ldots, \mathrm{B}=11$ ). The low middle and high registers are determined by the level III with values corresponding to sets $\{1,5,9\},\{2,3,4\}$ and $\{6,7$, $8\}$ respectively. The duration are provided by the fourth level by taking the eight-note as the minimal rhythmic subdivision and by associating the number 0 with the 'acciaccatura'. Dynamics are attached to the IX level as follows: $0=m f, 3=m p, 6=p p, 9=p$. The elements 7,10 , 1 and 4 correspond to rests. Finally, there are 4 ways of playing and they are related to the fourth level too, but with a different mapping, i.e., $\{0,1\}=$ vibrato, $\{3$, $4\}=$ normal, $\{6,7\}=$ 'al ponticello' et $\{9,10\}=$ tremolo. Figure 9 shows an extract of the piece with the numerical values associated with each level.

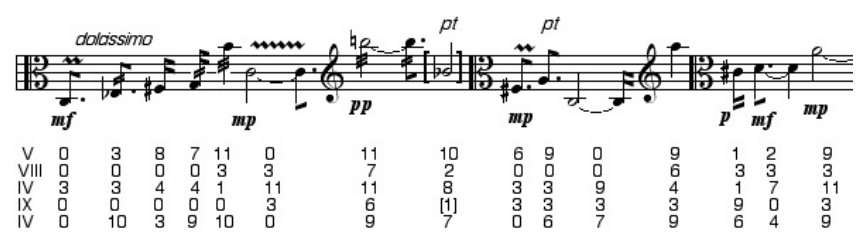

Fig. 9 A first extract of the piece Zone d'oubli

Figure 10 shows a further passage of the same piece where the previous parameters have been now associated with different levels. In particular, pitches are generated by the 6 -periodic sequence of the level II, while the low and high registers are given by the odd and even values of the level VI.

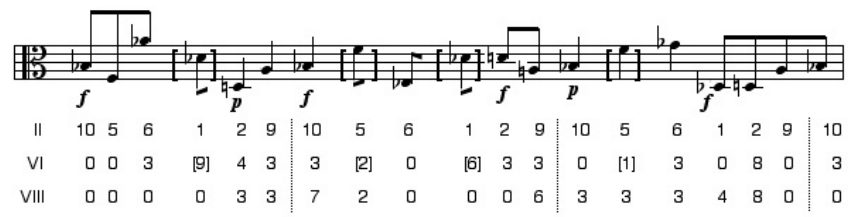

Fig. 10 An extract of the piece Zone d'oubli using a different mapping for musical parameters

Sometimes the generation of sequences by successive additions statistically increases the distribution of some particular values inside a given level. This remarkable phenomenon has been initially described by the composer in the case of successive additions from an initial sequence corresponding to Messiaen's second mode of limited transpositions. The intervallic structure of this mode corresponds to the 2 -periodic sequence $f=(2$ 1) and by successive additions, the composer generates several sequences, as shown in Appendix A. Levels I and II are generated by taking $c_{0}=11$ and $c_{1}=2$. For the third level the initial value $c_{2}=8$ is chosen and this gives a 16-periodic sequence. The next 8 levels, all starting with 8 , have a period 32 . There are two major properties that Vieru was not able to explain. Firstly, all levels are $2^{k}$-periodic for a given positive integer $k$. The second property has to do with the proliferation of values 8 and 4 at all levels excepts those which occur just before a change of period. This double phenomenon is still mysterious and, although we are not able to give a final explanation, we strongly believe that it has to do with the decomposition theorem. In fact, according to this theorem, the sequence $f=(21)$ can be decomposed (in a unique way) into the reducible component $f_{\text {red }}=\left(\begin{array}{l}69 \\ 9\end{array}\right)$ and into the reproducible component $f_{r e p}=\left(\begin{array}{ll}8 & 4\end{array}\right)$. Note that $f_{\text {red }}$ and $f_{\text {rep }}$ are sequences taking values in two subgroups isomorphic respectively to $\mathbf{Z}_{3}$ and $\mathbf{Z}_{4}$. More generally, by induction on $k$, every sequence $f$ such that $D^{k} f=(21)$ for a given integer $k$ and for which the various levels start with initial values $c_{i}$ for which $p\left(c_{i}\right)=8$ where $p$ is the projection on the subgroup isomorphic to $\mathbf{Z}_{3}$, can be decomposed into a sequence taking values in $\mathbf{Z}_{4}$ and the sequence (84). Notice that the values $c_{i}$ having this property are $8(=8+0), 11(=8+3), 2(=8+6)$ and $5(=8+9)$. In Vieru's example, the proliferation of 8 and 4 could be explained by the fact that starting from the third level, the initial values are always $c_{i}=8$. The proliferation of 4 and 8 in the growing process remains true if the initial values $c_{i}$ are such that $p\left(c_{i}\right)=8$ i.e., $c_{i}$ $=11,2$ or 5 . Some statistics are shown in Appendix B.

\section{Conclusion}

The finite difference calculus on periodic sequences taking values in a cyclic group gives rise to new interesting questions once it is approached from a compositional perspective. Starting from Anatol Vieru's original intuitions on the duality between sounds and intervals, we have shown some possible musical interpretations of the finite difference process (and its dual process, i.e., the finite additive process). We explain how reducible and reproducible sequences play the structural role of generators for any periodic sequence taking value in a cyclic group. The computational character of this fondamental decomposition result, which has been also integrated in a computer-aided compositional environment, suggests new possible musical applications of Vieru's original ideas. At the same time, it naturally leads to some generalizations whose musical relevance is still to be established.

\section{References}

1. C. Agon. OpenMusic: un langage visuel pour la composition musicale assistée par ordinateur. Thèse de doctorat, Paris VI, 1998.

2. M. Andreatta. Méthodes algébriques en musique et musicologie du XX siècle: aspects théoriques, analytiques et compositionnels. Thèse de doctorat, EHESS, IRCAM, 2003. 
3. M. Andreatta and D. T. Vuza. On some properties of periodic sequences in anatol vieru's modal theory. Tatra Mt. Math. Publ. 23, pages 1-15, 2001.

4. G. Balzano. The group-theoretic description of 12-fold and microtonal pitch systems. Computer Music Journal 4, pages $66-84,1980$.

5. D. Lewin. Generalized Musical Intervals and Transformations. Yale University Press, 1987.

6. G. Mazzola. Topos of Music. Birkhauser Verlag, 2003.

7. A. Vieru. Cartea modurilor, 1 (Le livre des modes, 1). Ed. Muzicala, Bucarest, 1980. Revised ed. The book of modes, 1993.

8. D. T. Vuza. Supplementary sets and regular complementary unending canons (in 4 parts). Perspectives of New Music, 29-31, 1991-93.
Appendix A. Proliferation of values 8 and 4

The finite addition process starts from the 2-periodic sequence $f=\left(\begin{array}{ll}2 & 1\end{array}\right)$ and with initial values equal to $c_{0}=11$, $c_{1}=2$ and $c_{i}=8$ for $i>1$. At each level we indicate the percentage of values 4 and 8 inside of the given period. Notice that at level 61 of period 128 , more than $90 \%$ of the elements belong to the set $\{4,8\}$. This percentage dramatically decreases in the following level which is the last one having period equal to 128 .

$$
\begin{array}{llllllll}
2 & 1 & 2 & 1 & 2 & 1 & 2 & 1
\end{array}
$$

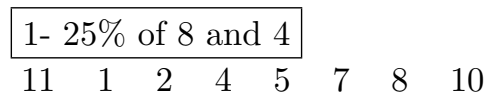

$$
\begin{array}{|lllllllll}
\hline 2-50 \% & \text { of } & 8 & \text { and } 4 & \\
\cline { 1 - 5 } & 2 & 1 & 2 & 4 & 8 & 1 & 8 & 4
\end{array}
$$

\begin{tabular}{|c|c|c|c|c|c|c|c|}
\hline \multicolumn{5}{|c|}{$3-25 \%$ of 8 and 4} & & \multirow[b]{2}{*}{1} & \multirow[b]{2}{*}{2} \\
\hline 8 & 10 & 11 & 1 & 5 & & & \\
\hline 2 & 4 & 5 & 7 & 11 & & 7 & 8 \\
\hline \multicolumn{5}{|c|}{$4-37.5 \%$ of 8 and 4} & \multirow{3}{*}{7} & & \multirow[b]{2}{*}{10} \\
\hline 8 & 4 & 2 & 1 & 2 & & 8 & \\
\hline 8 & 10 & 2 & 7 & 2 & & 8 & 4 \\
\hline \multicolumn{5}{|c|}{$5-50 \%$ of 8 and 4} & & & \\
\hline 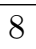 & 4 & 8 & 10 & 11 & & 1 & \\
\hline 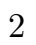 & 10 & 8 & 10 & - & & & \\
\hline
\end{tabular}

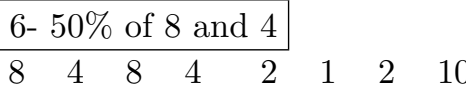

\begin{tabular}{|c|c|c|c|c|c|c|c|}
\hline \multicolumn{4}{|c|}{$7-25 \%$ of 8 and 4} & \multirow[b]{2}{*}{8} & \multirow[b]{2}{*}{10} & \multirow[b]{2}{*}{11} & \\
\hline 8 & 4 & 8 & 4 & & & & 1 \\
\hline 11 & 1 & 5 & 7 & 5 & 1 & 2 & 10 \\
\hline 2 & 10 & 2 & 10 & 2 & 4 & 5 & \\
\hline 5 & 7 & 11 & 1 & 11 & 7 & 8 & \\
\hline
\end{tabular}

$\begin{array}{llllllll}2 & 4 & 2 & 10 & 8 & 1 & 8 & 4\end{array}$

\begin{tabular}{|c|c|c|c|c|c|c|c|}
\hline \multicolumn{5}{|c|}{$8-37.5 \%$ of 8 and 4} & \multirow[b]{2}{*}{4} & \multirow[b]{2}{*}{2} & \multirow[b]{2}{*}{1} \\
\hline 8 & 4 & 8 & 4 & 8 & & & \\
\hline 2 & 1 & 2 & 7 & 2 & 7 & 8 & 10 \\
\hline 8 & 10 & 8 & 10 & 8 & 10 & 2 & 7 \\
\hline 2 & 7 & 2 & 1 & 2 & 1 & 8 & 4 \\
\hline \multicolumn{5}{|c|}{ 9- $50 \%$ of 8 and 4} & & & \\
\hline 8 & 4 & 8 & 4 & 8 & 4 & 8 & 10 \\
\hline 11 & 1 & 2 & 4 & 11 & 1 & 8 & 4 \\
\hline 2 & 10 & 8 & 4 & 2 & 10 & 8 & 10 \\
\hline 5 & 7 & 2 & 4 & 5 & 7 & 8 & 4 \\
\hline
\end{tabular}

\begin{tabular}{|c|c|c|c|c|c|c|}
\hline & 68 & $5 \%$ & of 8 & an & & \\
\hline 8 & 4 & 8 & 4 & 8 & 4 & \\
\hline 2 & 1 & 2 & 4 & 8 & 7 & \\
\hline 8 & 10 & 8 & 4 & 8 & 10 & \\
\hline 2 & 7 & 2 & & 8 & 1 & \\
\hline
\end{tabular}




\begin{tabular}{|c|c|c|c|c|c|c|c|}
\hline \multicolumn{6}{|c|}{$11-56.25 \%$ of 8 and 4} & \multirow[b]{2}{*}{4} & \multirow[b]{2}{*}{8} \\
\hline 8 & 4 & 8 & 4 & & & & \\
\hline 8 & 10 & 11 & 1 & & & 1 & 8 \\
\hline 8 & 4 & 2 & 1 & & & 10 & 8 \\
\hline 8 & 10 & 5 & 7 & & 1 & 7 & 8 \\
\hline \multicolumn{6}{|c|}{$12-68.75 \%$ of 8 and 4} & \multirow[b]{2}{*}{8} & \multirow[b]{2}{*}{4} \\
\hline 8 & 4 & 8 & 4 & 8 & 4 & & \\
\hline 8 & 4 & 2 & 1 & 2 & 7 & 8 & 4 \\
\hline 8 & 4 & 8 & 10 & 8 & 10 & 8 & 4 \\
\hline 8 & 4 & 2 & 7 & 2 & \multirow[t]{2}{*}{1} & \multirow[t]{2}{*}{8} & \multirow[t]{2}{*}{4} \\
\hline \multicolumn{5}{|c|}{$13-75 \%$ of 8 and 4} & & & \\
\hline 8 & 4 & 8 & 4 & 8 & 4 & 8 & 4 \\
\hline 8 & 4 & 8 & 10 & 11 & 1 & 8 & 4 \\
\hline 8 & 4 & 8 & 4 & 2 & 10 & 8 & 4 \\
\hline 8 & 4 & 8 & 10 & 5 & 7 & 8 & 4 \\
\hline \multicolumn{5}{|c|}{$14-50 \%$ of 8 and 4} & \multirow[b]{2}{*}{4} & \multirow[b]{2}{*}{8} & \\
\hline 8 & 4 & 8 & 4 & 8 & & & \\
\hline 8 & 4 & 8 & 4 & 2 & 1 & 2 & 10 \\
\hline 2 & 10 & 2 & 10 & 2 & 4 & 2 & 10 \\
\hline 2 & 10 & 2 & 10 & 8 & 1 & \multirow[t]{2}{*}{8} & \multirow[t]{2}{*}{4} \\
\hline \multicolumn{6}{|c|}{$16-37.5 \%$ of 8 and 4} & & \\
\hline 8 & 4 & 8 & 4 & 8 & 4 & 8 & 4 \\
\hline 8 & 4 & 8 & 4 & 8 & 4 & 2 & 1 \\
\hline 2 & 1 & 2 & 1 & 2 & 1 & 2 & 7 \\
\hline 2 & 7 & 2 & 7 & 2 & 7 & 8 & 10 \\
\hline 8 & 10 & 8 & 10 & 8 & 10 & 8 & 10 \\
\hline 8 & 10 & 8 & 10 & 8 & 10 & 2 & 7 \\
\hline 2 & 7 & 2 & 7 & 2 & 7 & 2 & 1 \\
\hline 2 & 1 & 2 & 1 & 2 & 1 & 8 & 4 \\
\hline
\end{tabular}

\begin{tabular}{|llllllll}
\hline 61 & $93.75 \%$ & of & 8 & and 4 & & \\
\cline { 1 - 5 } 8 & 4 & 8 & 4 & 8 & 4 & 8 & 4 \\
8 & 4 & 8 & 4 & 8 & 4 & 8 & 4 \\
8 & 4 & 8 & 4 & 8 & 4 & 8 & 4 \\
8 & 4 & 8 & 4 & 8 & 4 & 8 & 4 \\
8 & 4 & 8 & 4 & 8 & 4 & 8 & 4 \\
8 & 4 & 8 & 4 & 8 & 4 & 8 & 4 \\
8 & 4 & 8 & 4 & 8 & 4 & 8 & 4 \\
8 & 4 & 8 & 10 & 11 & 1 & 8 & 4 \\
8 & 4 & 8 & 4 & 8 & 4 & 8 & 4 \\
8 & 4 & 8 & 4 & 8 & 4 & 8 & 4 \\
8 & 4 & 8 & 4 & 8 & 4 & 8 & 4 \\
8 & 4 & 8 & 4 & 2 & 10 & 8 & 4 \\
8 & 4 & 8 & 4 & 8 & 4 & 8 & 4 \\
8 & 4 & 8 & 4 & 8 & 4 & 8 & 4 \\
8 & 4 & 8 & 4 & 8 & 4 & 8 & 4 \\
8 & 4 & 8 & 10 & 5 & 7 & 8 & 4
\end{tabular}

Appendix B. Percentage of elements 4 and 8 for different $c_{i}$

The following five figures show the graphics corresponding to the percentage of elements equal to 4 and 8 in the finite additive process corresponding to initial values $c_{i}$ equal to $8,2,5,11$ and 4 . In the first four cases, the graphics are very similar and they clearly show that the percentage of values 4 and 8 is globally increasing (although there is a local minimum appearing at each level preceding a change of period). This is no longer the case if the initial values $c_{i}$ are equal to 4 . In this case, as in the case of initial values $c_{i}$ different from $8,2,5$ or 11 , there is no proliferation of elements 4 and 8 in the finite addition process.

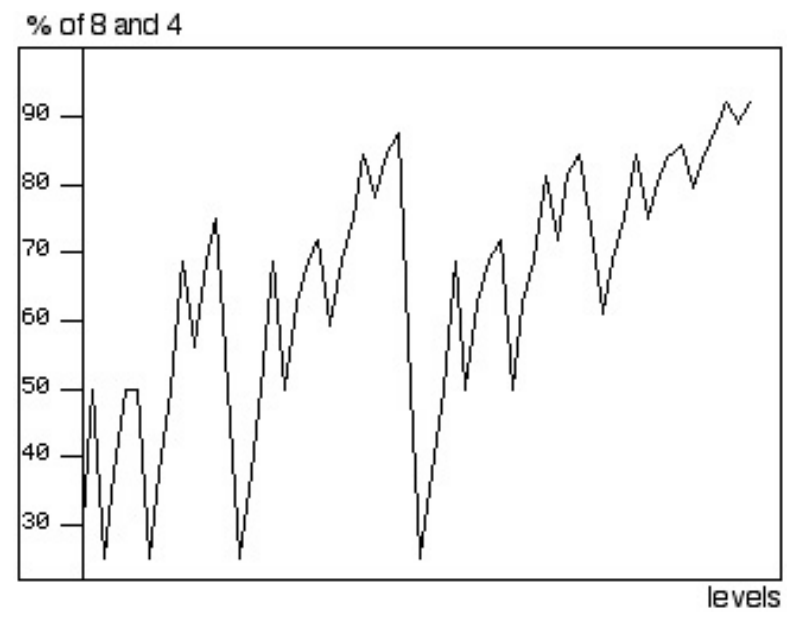

Fig. 11 Initial values equal to 8

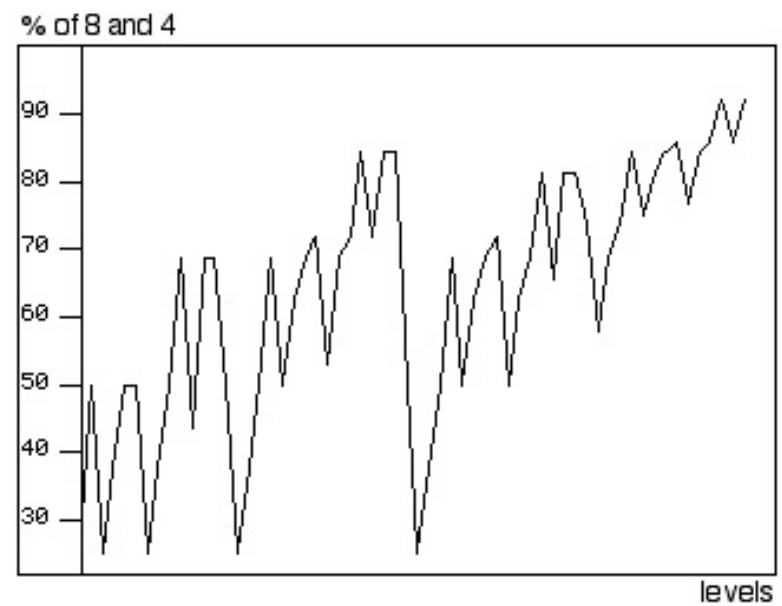

Fig. 12 Initial values equal to 2 


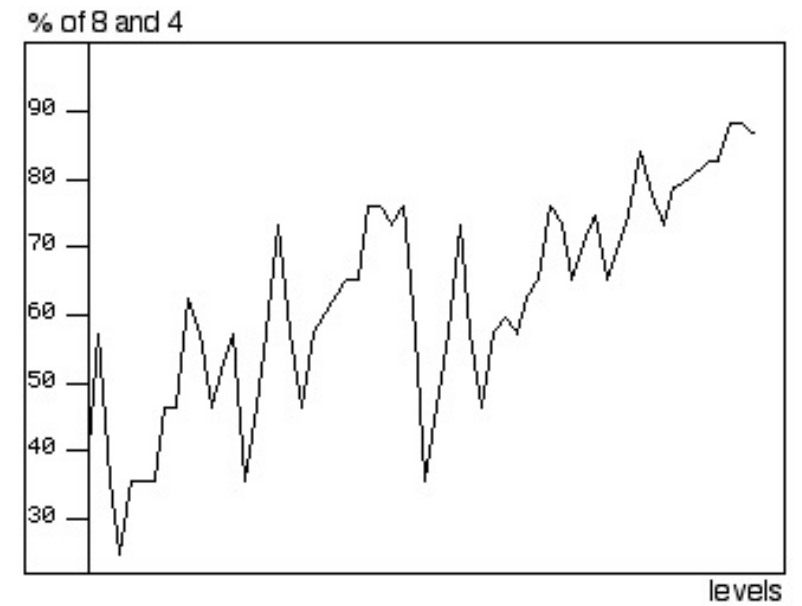

Fig. 13 Initial values equal to 5

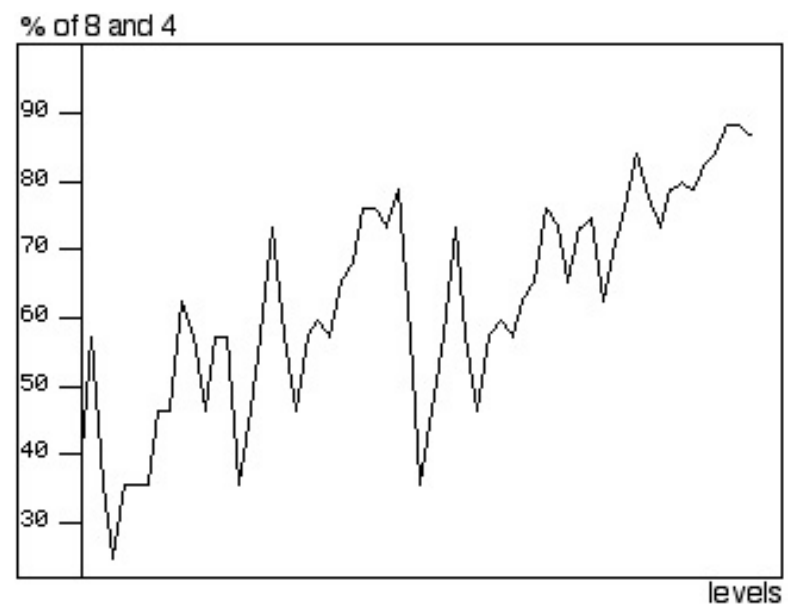

Fig. 14 Initial values equal to 11

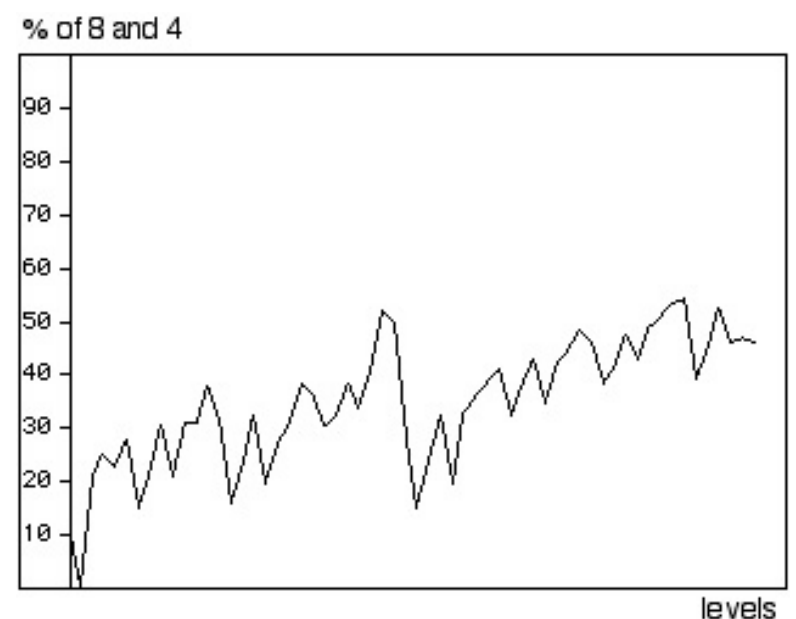

Fig. 15 Initial values equal to 4 